\title{
NOUVELle
}

\section{Altérations du PRC2 au sein des tumeurs malignes associées à la neurofibromatose de type 1 : des cellules de Schwann sans aucun complexe!}

Armelle Luscan ${ }^{1,2}$, Dominique Vidaud ${ }^{1,2}$, Nicolas Ortonne ${ }^{3}$, Pierre Wolkenstein ${ }^{4}$, Michel Vidaud ${ }^{1,2}$, Éric Pasmant ${ }^{1,2}$
${ }^{1}$ Service de biochimie et de génétique moléculaire, hôpital Cochin, AP-HP, Paris, France ;

${ }^{2}$ Inserm EA7331, université Paris Descartes,

Sorbonne Paris Cité, faculté des sciences pharmaceutiques et biologiques, 4, avenue de l'Observatoire, 75005 Paris,

France ;

${ }^{3}$ département de pathologie et université Paris Est Créteil (UPEC), hôpital Henri Mondor, Créteil, France ;

${ }^{4}$ département de dermatologie, centre de référence des neurofibromatoses, hôpital Henri Mondor, AP-HP, EA 4393 LIC, université Paris Est Créteil (UPEC), Créteil, France. eric.pasmant@gmail.com armelleluscan@gmail.com

\section{Les Rasopathies}

La voie de transduction intracellulaire des RAS-MAPK (mitogen-activated protein kinases) est une des voies le plus communément dérégulée en cancérologie [1]. Des mutations somatiques affectant cette voie de signalisation ont été décrites dans environ $30 \%$ des cancers humains. Dans les années 1990, la découverte de mutations constitutionnelles du gène NFI (neurofibromin 1) codant une protéine inhibitrice des protéines RAS, a montré pour la première fois que des dérégulations de cette voie de signalisation pouvaient aussi être à l'origine de maladies héréditaires du développement. Depuis, un ensemble de maladies héréditaires causées par des mutations constitutionnelles des acteurs de la voie RAS-MAPK ont été regroupées sous le nom de Rasopathies [2]. Ces maladies partagent des caractéristiques phénotypiques incluant, pour la majorité d'entre elles, une prédisposition tumorale.

\section{La neurofibromatose de type 1}

La neurofibromatose de type 1 ou NFl (MIM\#162200) est une Rasopathie causée par des mutations perte de fonction du gène suppresseur de tumeurs NFI. Plus de $90 \%$ des patients portent une mutation restreinte au gène $N F I$ et une importante variété de ces mutations perte de fonction ont été décrites [3]. Environ $10 \%$ des patients portent une grande délétion récurrente de plus de $\mathrm{l} \mathrm{Mb}$ incluant l'ensemble du gène NFI ainsi que 13 gènes adjacents [4]. Ces délétions récurrentes du locus NFI sont causées par des recombinaisons homologues non alléliques impliquant des séquences répétées paralogues situées de part et d'autre du gène NFI.

Vingt mille individus sont atteints de neurofibromatose de type 1 en France. Cette maladie est caractérisée par la présence de tumeurs bénignes des gaines nerveuses dénommées neurofibromes. Les neurofibromes sont constitués d'une prolifération de cellules de Schwann présentant une inactivation somatique du second allèle NF1. Certains des neurofibromes peuvent se transformer - si des événements génétiques additionnels surviennent - en tumeurs malignes des gaines nerveuses dénommées MPNST (malignant peripheral nerve sheath tumor). Ces MPNST représentent la première cause de mortalité des patients atteints de NFl.

\section{Du génotype au phénotype:} les grandes délétions du locus prédisposent aux MPNST

La NFl, comme d'autres maladies héréditaires de transmission dominante, est caractérisée par une expressivité clinique très variable. Dans le but d'identifier les facteurs génétiques contrôlant l'expression phénotypique de la maladie, une vaste banque de données génotype-phénotype consacrée à la NFl a été constituée à l'échelle de la France (centre de référence maladies rares des neurofibromatoses, Pr Pierre Wolkenstein).
Chez les patients présentant des mutations restreintes au gène NFI, l'étude des corrélations phénotypiques au sein de 275 familles multiplex atteintes de NFl a permis de montrer l'absence d'influence du type de mutation constitutionnelle sur la variabilité phénotypique [5]. La sévérité de la maladie ne peut pas être prédite par la nature ou la localisation de la mutation dans le gène NFI. Cependant, une étude d'association ciblée sur des données familiales (family-based association study, FBAT) a permis la première identification d'un modificateur génétique du développement des neurofibromes : le grand ARN non codant ANRIL (antisense non-coding RNA in the INK4 locus), localisé en 9p2l [6, 11] $(\rightarrow)$.

Pour les patients de $\varepsilon$. Pasmant et al. récurrentes de l'en- page 564

semble du locus NFI, nous avons confirmé l'existence d'un phénotype plus sévère que celui causé par les mutations restreintes au gène $N F 1$, avec notamment une susceptibilité accrue aux MPNST [4]. Cette observation suggérait donc l'existence d'un autre gène suppresseur de tumeur - outre NFI - parmi les 13 gènes situés dans l'intervalle de délétion récurrente.

Locus NF1 : un suppresseur de tumeurs peut en cacher un autre

Des données récentes faisaient du gène SUZ12 (suppressor of zeste 12 homolog) un excellent candidat parmi ces 13 gènes. 


\section{Génotype constitutionnel}

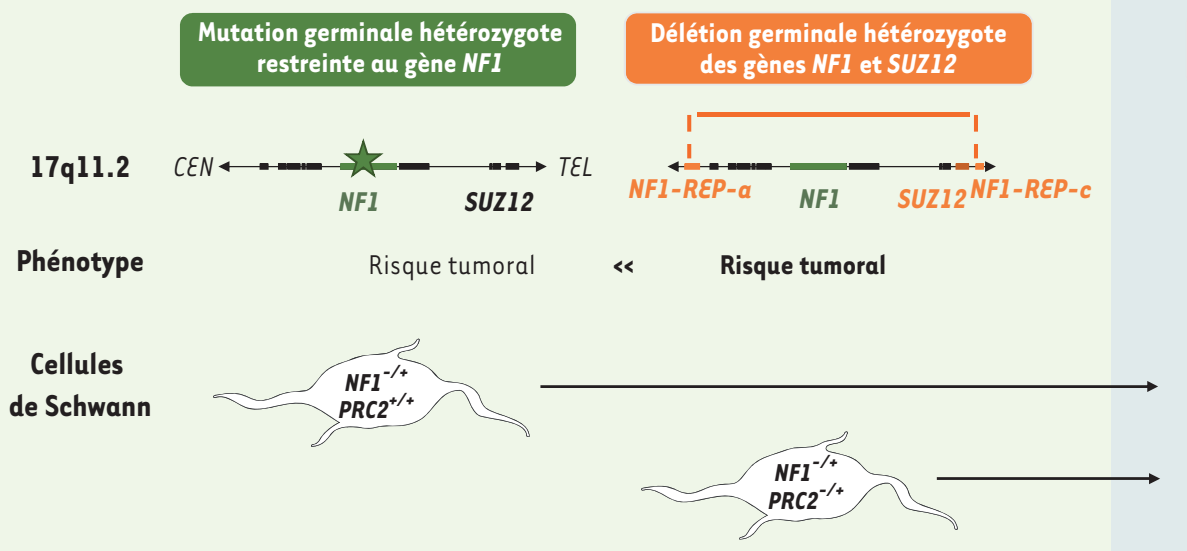

Génotype tumoral

Mutations et/ou délétions somatiques homozygotes de NFI et SUZ12

Événements génétiques additionnels

MPNST : tumeur maligne des gaines nerveuses périphériques

Figure 1. Les pertes de fonction de NF1 et SUZ12 coopèrent dans la tumorigenèse des MPNST associés à la NF1. Environ $10 \%$ des patients présentent de grandes délétions récurrentes de plus de $1 \mathrm{Mb}$ incluant l'ensemble du gène NFl ainsi que 13 gènes additionnels dont le gène SUZ12. Ces grandes délétions constitutionnelles du locus NFI sont associées à un risque important de survenue de tumeurs malignes des gaines nerveuses. Dans les MPNST, une inactivation du second allèle sauvage du gène NFI est observée. Une altération bi-allélique du gène SUZ12 est également retrouvée dans la majorité de ces tumeurs.

SUZ12 code une protéine majeure du complexe du groupe Polycomb 2 (polycomb repressive complex $2, \mathrm{PRC} 2)$. Ce complexe protéique est impliqué dans le remodelage de la chromatine parce qu'il induit une modification post-traductionnelle des histones. Le complexe PRC2 catalyse la triméthylation de la lysine 27 de l'histone H3. La modification épigénétique H3K27me3 est une marque chromatinienne associée à une répression transcriptionnelle [7]. De plus, l'interaction entre ANRIL et SUZ12 a été démontrée comme nécessaire à la répression transcriptionnelle épigénétique de certains gènes cibles.

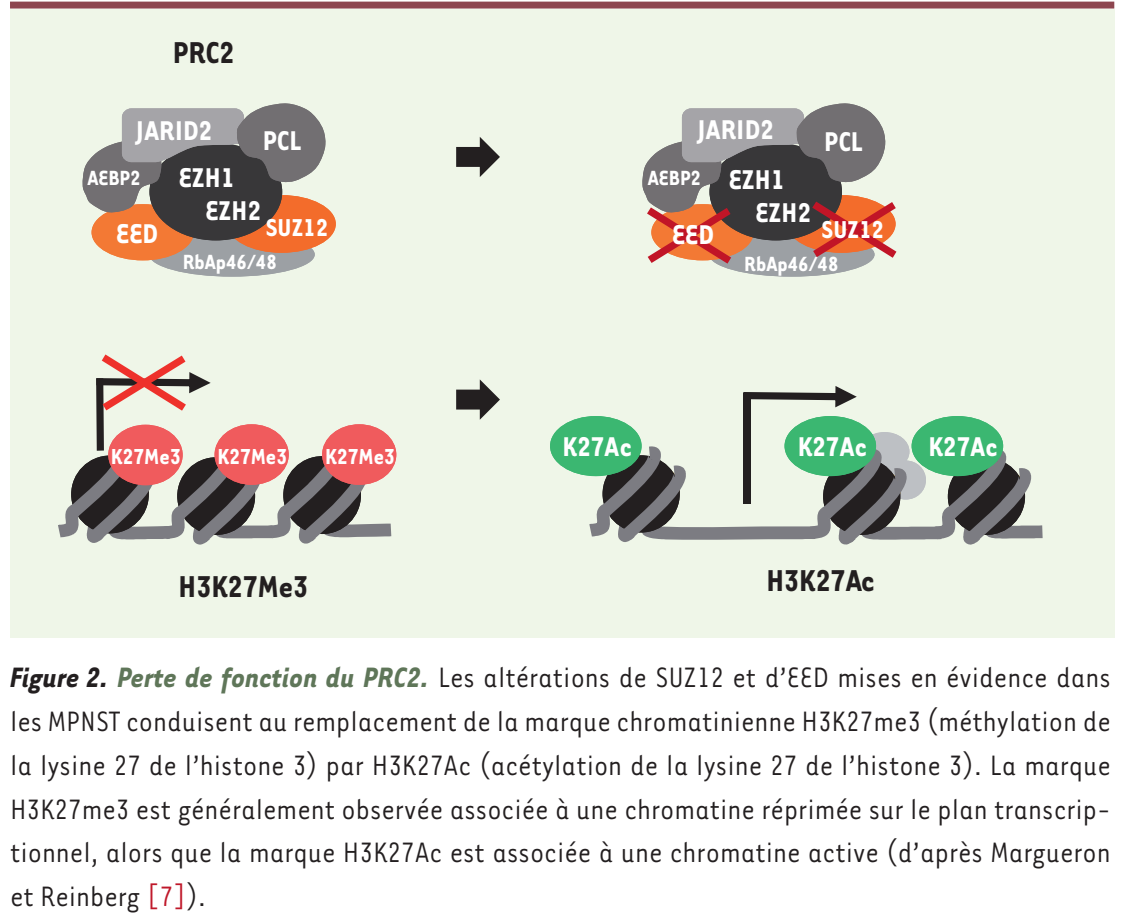

De manière intéressante, des mutations perte de fonction de composants du PRC2 ont été identifiées dans plusieurs types d'hémopathies malignes [8]. Des mutations tronquantes de la protéine عZH2 (enhancer of zeste homolog 2) portant l'activité catalytique méthyltransférase du PRC2, ainsi que des mutations de SUZ12, ont été décrites.

\section{Des cellules de Schwann}

sans aucun complexe

L'étude ciblée par séquençage nouvelle génération (NGS, next generation sequencing; plate-forme de NGS de I'hôpital Cochin, Paris) de 13 gènes impliqués dans l'établissement de la marque H3K27me3 (dont SUZ12) a été menée pour un panel de 51 MPNST, constitué dans le cadre d'une collaboration internationale [9]. Une étude pangénomique par CGH (comparative genomic hybridization)-array a également été réalisée. Ces approches génomiques ont permis de mettre en évidence une inactivation totale du gène SUZ12, ou plus rarement du gène $\varepsilon \varepsilon D$ (embryonic ectoderm development), dans plus de la moitié des MPNST. La protéine $\varepsilon \varepsilon D$, comme SUZ12, fait partie du complexe 
minimal PRC2. La perte de la marque épigénétique H3K27me3 a été confirmée par l'étude immunohistochimique de coupes des tumeurs MPNST mutées. Pour la première fois, l'implication majeure du PRC2 était montrée dans la tumorigenèse associée à la NFl (Figure 1).

Les chaises musicales:

\section{H3K27Ac remplace H3K27me3}

Un modèle murin de double invalidation hétérozygote en cis $\mathrm{Nfl}^{+/-}$;Suz12 ${ }^{+/-}$a permis de confirmer l'importance de la coopération entre les altérations de NFI et de SUZ12 dans la tumorigenèse des MPNST [9]. L'inactivation du PRC2 entraîne la perte de la marque chromatinienne H3K27me3 au sein des tumeurs. Une acétylation de la même lysine est également observée en lieu et place de la méthylation manquante (Figure 2). La marque épigénétique H3K27Ac est en général retrouvée associée à une chromatine active sur le plan transcriptionnel. Cette acétylation conduit au recrutement de protéines à bromodomaines, lectrices de la marque H3K27Ac et associées à des facteurs de transcription. L'analyse des données de transcriptome et d'immunoprécipitation de la chromatine couplée au NGS (chromatin immunoprecipitation (hIP-sequencing) acquises dans les tumeurs mutées a permis de proposer des cibles transcriptionnelles de ces modifications chromatiniennes. Les altérations du PRC2 permettraient notamment une amplification de la signature transcriptionnelle associée à l'activation de la voie RAS-MAPK.

\section{H3K27me3 et H3K27Ac : biomarqueurs} de mutation, voire de sensibilité thérapeutique

Des inhibiteurs de la lecture de la marque H3K27Ac récemment développés ont montré leur efficacité antitumorale in vivo dans le modèle murin préclinique transgénique $\mathrm{NFI}^{+/-} ; \mathrm{Suzl} 2^{+/-}$en association à une thérapie ciblée de la voie RAS-MAPK (anti-MEK) [9]. Ces inhibiteurs de bromodomaines [10] représentent donc une stratégie thérapeutique prometteuse pour les MPNST associées à la NFl, qui sont des tumeurs malignes très agressives.

Le gène NFI a récemment été décrit comme une des cibles somatiques les plus fréquemment mutées en cancérologie (voir les données en ligne du projet the cancer genome atlas incluant plus de 3000 tumeurs de 12 types différents). Au-delà de la NFl et des maladies rares, ce modèle de coopération entre les anomalies somatiques de la voie RAS-MAPK et du PRC2 pourrait ainsi s'appliquer à d'autres types tumoraux sporadiques, qui se révèleraient alors potentiellement sensibles à ces nouvelles épithérapies. $\diamond$ PRC2 alterations in NF1-associated malignant peripheral nerve sheath tumors: Schwann cells with no complex

\section{LIENS D'INTÉRÊT}

Les auteurs déclarent n'avoir aucun lien d'intérêt concernant les données publiées dans cet article.

\section{RÉFÉRENCES}

1. Schubbert S, Shannon K, Bollag G. Hyperactive Ras in developmental disorders and cancer. Nat Rev Cancer $2007 ; 7: 295-308$.

2. Bentires-Alj M, Kontaridis MI, Neel BG. Stops along the RAS pathway in human genetic disease. Nat Med $2006 ; 12: 283-5$.

3. Sabbagh A, Pasmant $\varepsilon$, Imbard A, et al. NFI molecular characterization and neurofibromatosis type I genotype-phenotype correlation: the French experience. Hum Mutat $2013 ; 34: 1510-8$.

4. Pasmant $\varepsilon$, Sabbagh A, Spurlock G, et al. NFl microdeletions in neurofibromatosis type 1 : from genotype to phenotype. Hum Mutat 2010 ; 31 : E1506-18.

5. Sabbagh A, Pasmant $\varepsilon$, Laurendeau I, et al. Unravelling the genetic basis of variable clinical expression in neurofibromatosis 1 . Hum Mol Genet $2009 ; 18: 2768-78$.

6. Pasmant $\varepsilon$, Sabbagh A, Masliah-Planchon J, et al. Role of noncoding RNA ANRIL in genesis of plexiform neurofibromas in neurofibromatosis type 1. J Natl Cancer Inst 2011 ; 103 : 1713-22.

7. Margueron R, Reinberg D. The Polycomb complex PRC2 and its mark in life. Nature $2011 ; 469$ : 343-9.

8. Ntziachristos P, Tsirigos A, Van Vlierberghe P, et al. Genetic inactivation of the polycomb repressive complex 2 in T cell acute lymphoblastic leukemia. Nat Med 2012 ; $18: 298-301$.

9. De Raedt T, Beert $\varepsilon$, Pasmant $\varepsilon$, et al. PRC2 loss amplifies Ras-driven transcription and sensitizes cancers to epigenetic-based therapies. Nature 2014 (sous presse).

10. Filippakopoulos $P$, Knapp S. Targeting bromodomains: epigenetic readers of lysine acetylation. Nat Rev Drug Discov $2014 ; 13$ : 337-56.

11. Pasmant $\varepsilon$, Laurendeau I, Sabbagh A, et al. ANRIL ou l'étrange histoire d'un grand ARN non codant. Med Sci (Paris) $2010 ; 26: 564-6$.

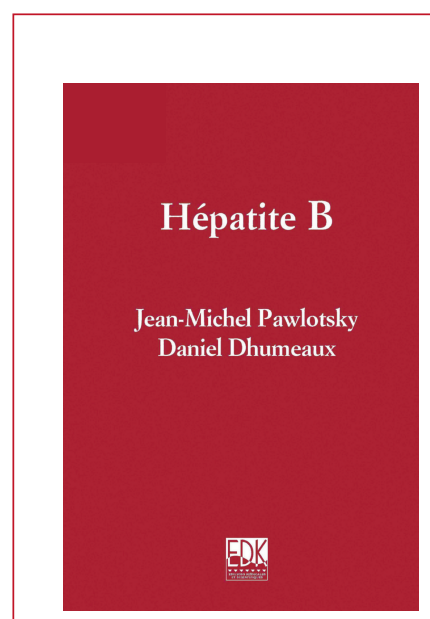

ISBN : 978-2-8425-4131-6 576 pages

\section{Bon de commande}

À retourner à EDK, 109, avenue Aristide Briand - 92541 Montrouge Cedex

Tél. : 0141177405 - Fax : 0155641394 - E-mail : edk@edk.fr

NOM : Prénom :

Adresse :

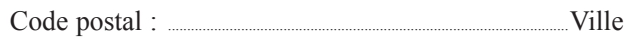

Pays :

Fonction :

Je souhaite recevoir l'ouvrage Hépatite B : $54 €+3 €$ de port $=\mathbf{5 7} €$ TTC offre exceptionnelle réservée aux abonnés à m/s jusqu'au 31 décembre 2014

en ................ exemplaire, soit un total de .................................... €

$\square$ Par chèque, à l'ordre de $\mathbf{E} \mathbf{D} \mathbf{K}$

$\square$ Par carte bancaire : $\square$ Visa $\square$ Eurocard/Mastercard

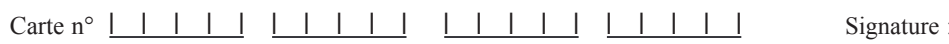

Date d'expiration: $\quad|\quad| \quad|\quad|$

$\mathrm{N}^{\circ}$ de contrôle au dos de la carte : $\quad|\quad| \quad \mid$ 